\title{
Direction of the microjet produced by the collapse of a cavitation bubble located in a corner of a wall and a free surface
}

\author{
Akihito Kiyama (1) \\ Institute of Global Innovation Research, Tokyo University of Agriculture and Technology, \\ Tokyo 184-8588, Japan \\ and Department of Mechanical and Aerospace Engineering, Utah State University, \\ Logan, Utah 84322, USA
}

Takaaki Shimazaki@

Department of Mechanical Systems Engineering, Tokyo University of Agriculture and Technology, Tokyo 184-8588, Japan

José Manuel Gordillo

Área de Mecánica de Fluidos, Departamento de Ingeniería Aeroespacial y Mecánica de Fluidos, Universidad de Sevilla, Avenida de los Descubrimientos s/n 41092, Sevilla, Spain

Yoshiyuki Tagawa $\odot^{*}$

Department of Mechanical Systems Engineering, Tokyo University of Agriculture and Technology, Tokyo 184-8588, Japan

(Received 11 November 2020; accepted 8 July 2021; published 5 August 2021)

In this paper, we present a simplified theoretical model based on the method of images that predicts the direction of the microjet produced after the implosion of a cavitation bubble created in a corner of a free interface and rigid wall. Our theoretical predictions have been verified by means of a thorough experimental study in which the distances of the pulsed-laser cavitation bubble to the wall and the free surface are varied in a systematic manner. In addition, we extend the predictions to arbitrary values of the corner angle, $\pi /(2 n)$ with $n$ a natural number. The present analytical solution might be a hint to a practical design for preventing cavitation-induced damage.

DOI: 10.1103/PhysRevFluids.6.083601

\section{INTRODUCTION}

Cavitation bubbles can form a microjet directed towards a rigid wall when the collapse takes place near the solid surface [1]. The impingement of the microjet on the solid boundary is one of the sources of cavitation-induced damage (e.g., Ref. [2]). A number of contributions have been devoted to predict and analyze the direction of the high-speed microjet with different geometries or properties, such as a rigid flat surface [3], a rigid curved surface [4], an ice surface [5], an elastic surface [6], a composite surface [7] and a free surface [8], and they all reveal that the jet direction is largely affected by the rigidity and the geometry of the flow boundaries.

Kucera and Blake [9] have studied the collapse of a cavitation bubble placed at the corner of two solid substrates forming an angle of either $\alpha=\pi / 2$ or $\alpha=\pi / 4$. Brujan et al. [10] experimentally

*tagawayo@cc.tuat.ac.jp 

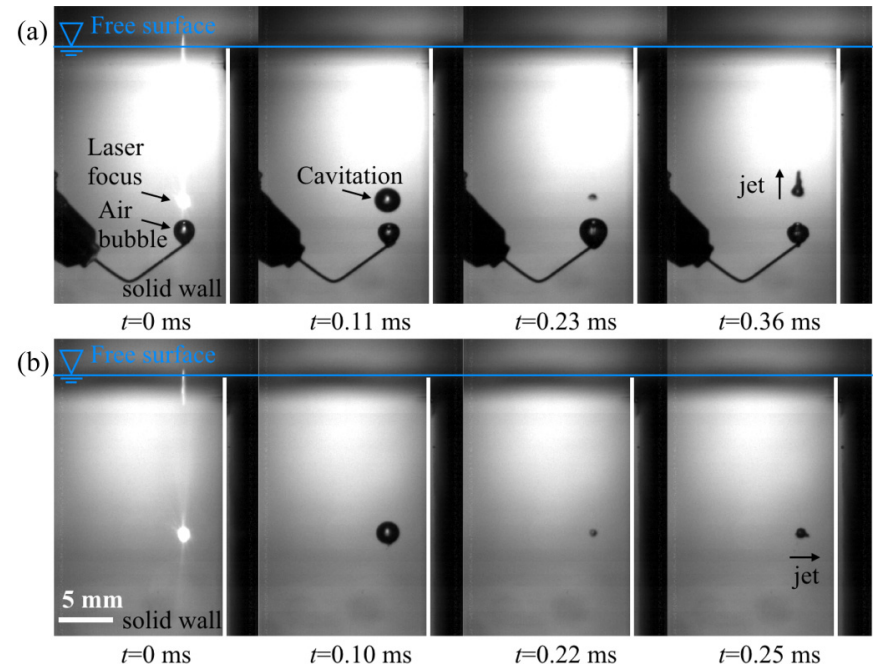

FIG. 1. High-speed images of a collapsing bubble with a maximum diameter $D$ in the vicinity of a solid surface and of an air bubble (a) and that in the absence of the air bubble (b). The two cases shown have similar values of the ratios $w / D$ and $h / D$, with $w$ and $h$ denoting, respectively, the distances of the center of the cavitation bubble to the wall and the free surface.

showed the direction of the microjet at the corner of two solid plates $(\alpha=\pi / 2)$ is proportional to the ratio of distances from the plates to the bubble. Tagawa and Peters [11] employed the method of images for solving the case $\alpha=\pi / n$, where $n$ is a natural number, and obtained analytical solutions for the jet direction that were confirmed experimentally. The method of images has been also adopted to analyze the motion of a bubble parallelly sandwiched by both a free surface and solid bottom [12]. Recently, Wang et al. [13] conducted numerical simulations that show a fair agreement with the published data for $\alpha=\pi / 2$ [10] and their experiments for $\alpha=\pi / 4$. The microjet direction inside the confined geometries $[14,15]$ and that near the slotlike structured surface [16] have also been studied.

In applications, it is desirable to prevent the impingement of the jet over the solid since, in this way, the lifetime of different types of fluid machinery, such as ship propellers, can be substantially increased [17]. An approach to achieve this is the installation of another surface with a different impedance. Luo et al. [18] placed an air bubble right next to the cavitation bubble and found that the direction of bubble movement upon its collapse was altered. Indeed, our preliminary observation [Fig. 1(a)] shows that the jet produced by the implosion of a laser-induced cavitation bubble generated in the vicinity of an air bubble is directed away from it. More recently, Avila et al. [19] demonstrated that a solid wall covered with micron-sized gas bubbles can alter the jet direction and help prevent the collision of the jet. However, to our knowledge, the theoretical prediction of the microjet direction in a corner of both free and rigid surfaces has not yet been provided.

Here, we provide a theoretical prediction of the microjet direction in a corner of a wall and free surface as sketched in Fig. 2, which contains the major physical ingredients behind the control of the direction of the microjet by means of the addition of air bubbles near the collapsing vapor bubble. Making use of ideas in a previous contribution [11], we employ the potential flow approximation and theoretically derive the direction of the jet as a function of the bubble location (Sec. II). To make the derivation as simple as possible and without loss of generality, this model is particularized for the simplest case where the corner angle between a wall and free surface $\alpha=\pi / 2\left(=90^{\circ}\right)$. Our analytical predictions are compared with the results of a systematic experimental study in which a laser-induced cavitation bubble is generated at well-defined distances from the wall and the free surface (Secs. III and IV). In addition, we derive a generalized model for the jet angle $\beta$ for corner angles $\alpha=\pi /(2 n)$ with $n$ a natural number (Sec. V). 


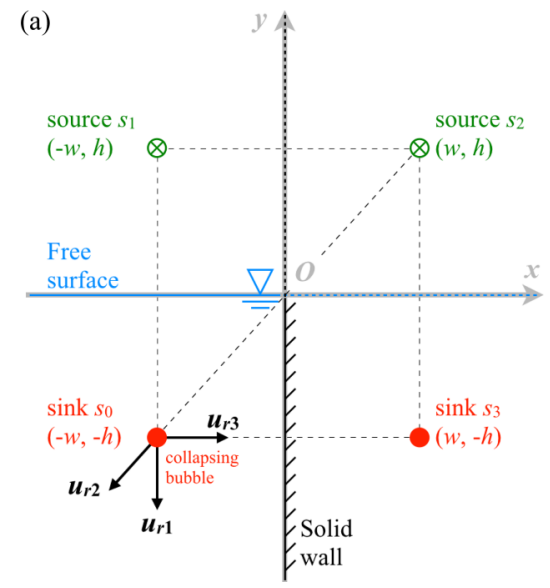

(b)

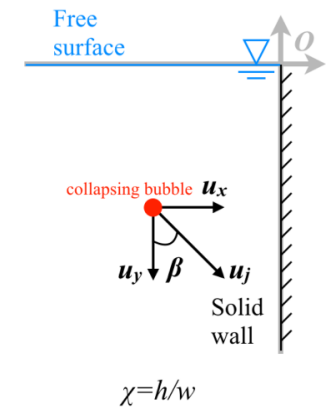

FIG. 2. (a) The schematic of the system on $x-y$ coordinates marked in gray. Green markers $(\otimes)$ and red markers $(\bigcirc)$ respectively represent the sources $\left(s_{1}\right.$ and $\left.s_{2}\right)$ and sinks $\left(s_{0}\right.$ and $\left.s_{3}\right)$. The velocity vectors affecting the bubble induced from mirrored images are denoted as $u_{r 1}, u_{r 2}$, and $u_{r 3}$. (b) The illustration of the microjet direction $\beta$. The horizontal $\left(u_{x}\right)$ and vertical $\left(u_{y}\right)$ components of the velocity vector acting on the bubble $u_{j}$ are denoted.

\section{THEORY}

A flow field induced by a spherically collapsing bubble is obtained by considering a sink [20] [Fig. 2(a), $s_{0}$ at $(-w,-h)$ ]. A solid wall and free surface are respectively modeled by placing the sink [Fig. 2(a), $s_{3}$ at $(w,-h)$ ] and source [Fig. 2(a), $s_{1}$ at $(-w, h)$ ] [21]. This sink-source system is appropriate since the bubble expansion does not significantly deform the boundaries in our experiments. Noteworthy, another source $s_{2}$ must be placed at $(w, h)$ to satisfy the boundary conditions as Tagawa and Peters [11] found the microjet direction $\beta$ [Fig. 2(b)] could not be modeled by simply considering the superposition of only two mirror images.

We now derive the microjet direction $\beta$. The radial speed $u_{r}$ at a certain distance $R$ from the sink/source is generally described as $u_{r}=Q /\left(4 \pi R^{2}\right)$, where $Q$ is the flow rate. The magnitudes of the velocity vectors at the bubble location $u_{r 1}, u_{r 2}$, and $u_{r 3}$ originated from respectively the sink/source $s_{1}, s_{2}$, and $s_{3}$ are

$$
u_{r 1}=\frac{Q}{16 \pi h^{2}}, \quad u_{r 2}=\frac{Q}{16 \pi\left(h^{2}+w^{2}\right)}, \quad u_{r 3}=\frac{Q}{16 \pi w^{2}} .
$$

The microjet direction $\beta$ can be calculated as

$$
\beta=\arctan \frac{u_{x}}{u_{y}}=\arctan \frac{1-\left(1+\chi^{2}\right)^{-3 / 2}}{\chi^{-2}+\chi\left(1+\chi^{2}\right)^{-3 / 2}},
$$

where $u_{x}$ and $u_{y}$ are the magnitudes of both the horizontal and the vertical components of the flow velocity vector as a function of $u_{r 1}, u_{r 2}, u_{r 3}$ and the dimensionless bubble location parameter $\chi=$ $h / w$ expressed as

$$
\begin{aligned}
& u_{x}=u_{r 3}-u_{r 2} \frac{w}{\sqrt{h^{2}+w^{2}}}=\frac{Q}{16 \pi w^{2}}\left[1-\frac{1}{\left(1+\chi^{2}\right)^{3 / 2}}\right], \\
& u_{y}=u_{r 1}+u_{r 2} \frac{h}{\sqrt{h^{2}+w^{2}}}=\frac{Q}{16 \pi w^{2}}\left[\frac{1}{\chi^{2}}+\frac{\chi}{\left(1+\chi^{2}\right)^{3 / 2}}\right] .
\end{aligned}
$$

Equation (2) indicates that the direction of the microjet only depends on the dimensionless bubble location $\chi$. For extreme conditions, the model predicts $\lim _{\chi \rightarrow \infty} \beta=90^{\circ}$ and $\lim _{\chi \rightarrow 0} \beta=0^{\circ}$. We 
(a)
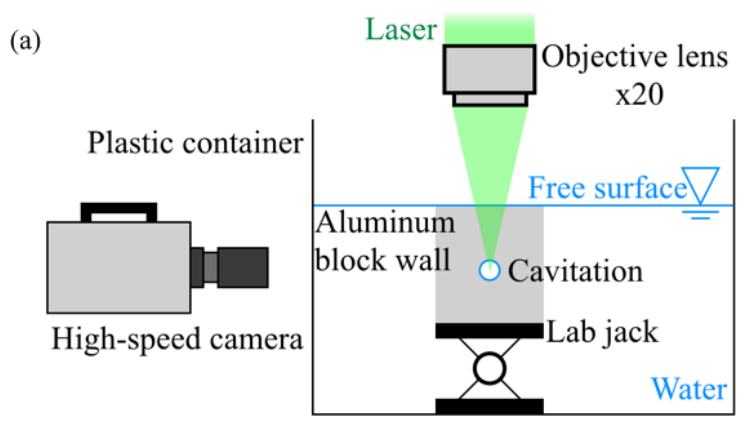

(b)
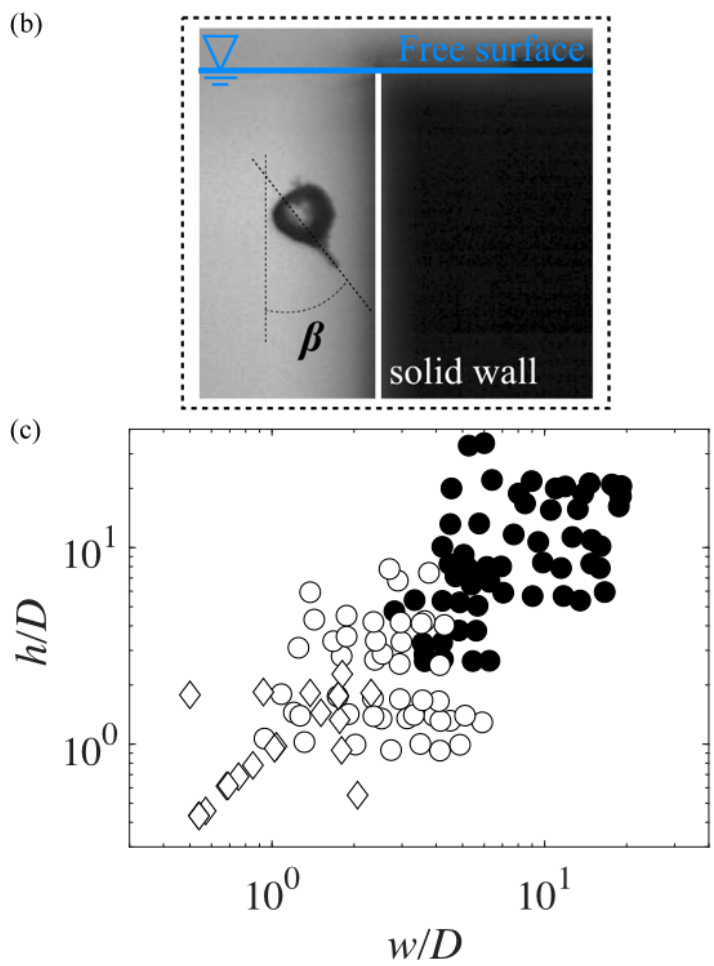

FIG. 3. (a) Experimental setup (not to scale). An aluminum block is used as a wall. The edge of the aluminum block reaches the free surface. A high-speed camera films the laser-induced cavitation bubble by using the backlight method. (b) An example image of a bubble with the illustration of the angle $\beta$. (c) Parameter space, where $h / D=0$ and $w / D=0$ respectively indicate a free surface and wall. Circles show the data taken from the series of experiments with diameters $D$ up to $3.6 \mathrm{~mm}$, where the overall bubble shape was mostly spherical. Data taken from the series of experiments with the larger diameter $D$ are marked by diamonds. Most of those diamonds are located at smaller $h / D$ or $w / D$ than the circles, where the bubble often leads to its nonspherical growth or a noticeable deformation of the free surface.

note that in contrast with the case of two rigid walls, where $\beta=45^{\circ}$ for $\chi=1$ [11], in the case at hand the deflection angle is $\beta=45^{\circ}$ for $\chi \simeq 1.38$ by virtue of Eq. (2).

\section{EXPERIMENTAL SETUP}

Figure 3(a) shows the experimental setup we used. A pulsed laser beam (Nd:YAG laser Nano S PIV, Litron Lasers Ltd., UK, wavelength $532 \mathrm{~nm}$, pulse duration $6 \mathrm{~ns}$ ) passes through an objective 
lens [MPLN series, Olympus Co., magnification 20 times, numerical aperture (NA) value 0.25] and illuminates a point inside ultrapurified water (produced by Milli-Q) inside an acrylic container (130 $\mathrm{mm} \times 165 \mathrm{~mm} \times 127 \mathrm{~mm}$ ). A cavitation bubble emerges and expands. A microjet then forms when the bubble collapses near the surface. For details of the laser and optical components, see Ref. [22].

A high-speed camera (FASTCAM SA-X, Photron Co.) is synchronized with the laser pulse through a delay generator (model 575 Pulse/Delay Generator, BMC). The typical resolutions of recording were set at $20000-100000 \mathrm{fps}$ and $0.1-0.2 \mathrm{~mm} /$ pixel. We controlled the maximum bubble diameter $D$, and the bubble location $(\chi=h / w)$, and measured the direction of the microjet $\beta$ [see Fig. 3(b)]. Experiments are repeated five times under each condition.

We first varied the location of a cavitation bubble ( $h$ and $w$ ). The bubble diameters are up to $D=3.6 \mathrm{~mm}$. The laser light was introduced vertically from the free surface. We observed the intermediate jet [open circles in Fig. 3(c), $D=2.98 \pm 0.46 \mathrm{~mm}$ ] and the weak jet [solid circles in Fig. 3(c), $D=1.39 \pm 0.98 \mathrm{~mm}$ ] based on a classification [23]. Our experiments reveal that bubbles collapse preserving the spherical symmetry until the very end, when a jet is issued. We only analyzed the experimental data corresponding to the intermediate-jet regime because the jet is stronger, causes more severe damage, and it is much easier to identify experimentally. The weak jet was observed when the bubble is located far enough from the surfaces, or the bubble diameter is small enough [Fig. 3(c)], as expected.

We then performed two series of experiments with bubbles of diameters $D \leqslant 4.7 \mathrm{~mm}$, where the laser light was introduced horizontally through the container wall. We first varied both $h$ and $w$ from 2 to $10 \mathrm{~mm}$ (corresponding $\chi=h / w$ ranges from 0.3 to 3.5 ) at larger cavitation diameters $D=4.35 \pm 0.19 \mathrm{~mm}$. We then placed a cavitation bubble at the locations $h \sim w \sim 2 \mathrm{~mm}$ and $h \sim$ $w \sim 3 \mathrm{~mm}$ ( $\chi \sim 0.9$ for both conditions), and varied the cavitation diameter $D$ from 2.0 to $4.7 \mathrm{~mm}$. We note that a relatively large diameter $D$, or the relatively small distance to one surface (i.e., the small $h$ or $w$ ), can introduce nonspherical bubble growth as well as a complex shape of the jet (see Sec. IV). In our experiments, nonspherical bubble growth, a noticeable deformation of the free surface, or the onset of cavities at the surfaces could be observed when $h / D \leqslant 1.5$ or $w / D \leqslant$ 1.5. Rigorously speaking, our model does not target these phenomena. Thus, the model does not necessarily predict the direction of the jet in such conditions. However, it is interesting to discuss these regimes especially from a practical point of view. We therefore included these experimental data and marked them with diamonds in Fig. 5.

\section{RESULTS}

The typical behaviors of the smaller cavitation bubble near the surfaces are shown in Fig. 4. The microjet caused by the collapse of a bubble located near the wall $(\chi \simeq 4.27)$ is directed towards the solid substrate, with $\beta \simeq 81^{\circ}$. In contrast, the bubble situated near the free surface $(\chi \simeq 0.23)$ produces a nearly vertical jet, with $\beta \simeq 1^{\circ}$. When the bubble is in a corner of the rigid wall and free surface, with $\chi \simeq 1.5$, the deflection angle is $\beta \simeq 45^{\circ}$.

Figure 5 summarizes the experimental data and compares the measured jet angle $\beta$ vs $\chi=h / w$ with the values predicted by Eq. (2), finding a good agreement between observations and predictions. The solid line denotes the prediction [Eq. (2)], while the circles and diamonds represent experimental results (Fig. 3). Overall, most of the data collapse into the solid line, which is independent of the bubble diameters tested herein. For a majority of data points, the difference between the measured angle and that estimated by the model fell within the range $\pm 10^{\circ}$. Our model, which is a function of only the bubble location parameter $\chi=h / w$, predicts the general trend of the microjet direction $\beta$ that occurs upon the collapse of a cavitation bubble affected by both the free surface and the wall. Interestingly, the data marked by the diamonds, which is often accompanied by nonspherical bubble growth or a noticeable free-surface deformation, largely lies within the range of $\pm 10^{\circ}$ as well.

We further discuss the influence of cavitation diameter $D$. Figure 6 summarizes the cavitation behavior with different $h(2 \mathrm{~mm} \leqslant h \leqslant 10 \mathrm{~mm}$ at fixed $w=8 \mathrm{~mm})$ and $w(2 \mathrm{~mm} \leqslant w \leqslant 10 \mathrm{~mm}$ 


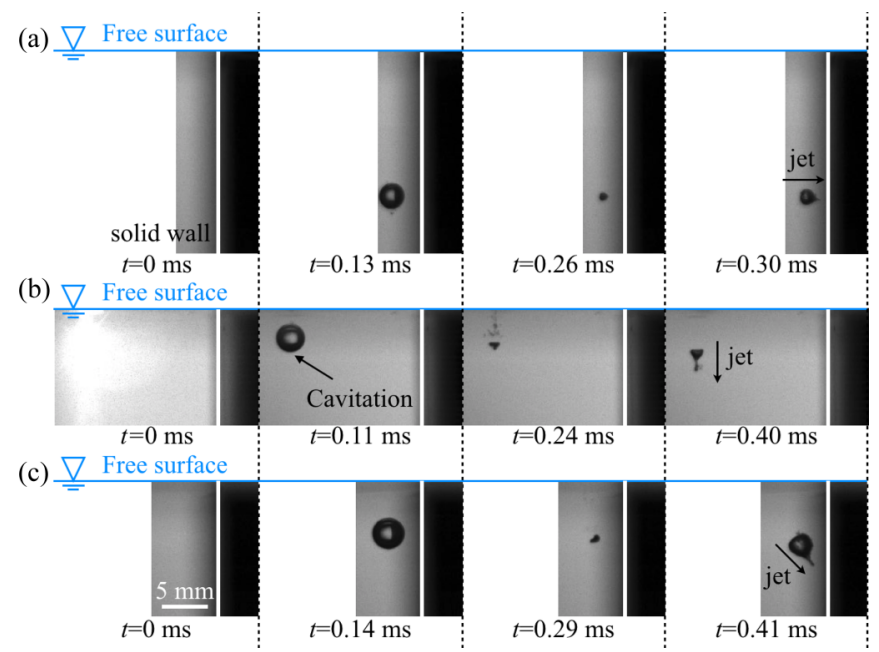

FIG. 4. High-speed images of collapsing cavitation bubbles. (a) The microjet goes towards the solid wall ( $\beta \simeq 81^{\circ}$ ) where $\chi \simeq 4.27$. (b) $\beta \simeq 1^{\circ}$ for $\chi \simeq 0.23$. (c) $\beta \simeq 45^{\circ}$ for $\chi \simeq 1.51$. The solid wall is on the righthand side of the bubble.

at fixed $h=8 \mathrm{~mm}$ ) values. The averaged diameter $D$ in this series of experiments was $D=$ $4.35 \pm 0.19 \mathrm{~mm}$ and thus significantly larger than that presented in Fig. 4. The relatively small distance from the boundaries, either $h / D$ or $w / D$, leads to nonspherical bubble growth [Figs. 6(a)$6(d)]$ as well as vertical jet formation above the free surface [Fig. 6(a)], free-surface deformation [Fig. 6(b)], and cavity inception at the wall surface [Fig. 6(c)]. Interestingly, we did not observe much of a discrepancy between the experimental data and model even in these cases (see Fig. 5). We conjecture that the situations of cavitation bubble dynamics in these cases were similar to that near the single boundary as the distance to one surface, either $h / D$ or $w / D$, gets smaller [e.g., Figs. 6(a) and 6(c)]. It is probably the reason why the model describes the data even though the bubble causes the phenomena, which the model does not take into account.

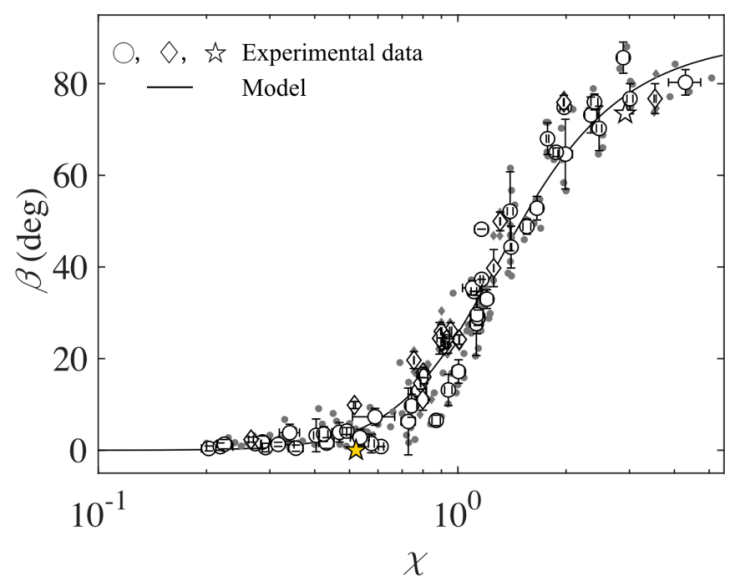

FIG. 5. A comparison with the experimental data (circles, diamonds, and stars) and the model [black line, Eq. (2)] as a function of $\chi$. The larger markers (circles and diamonds) show the mean value calculated by the multiple runs disclosed by the small gray markers. The error bars represent the standard deviations estimated from multiple runs if possible. The solid and open stars represent the values estimated from Fig. 1. 


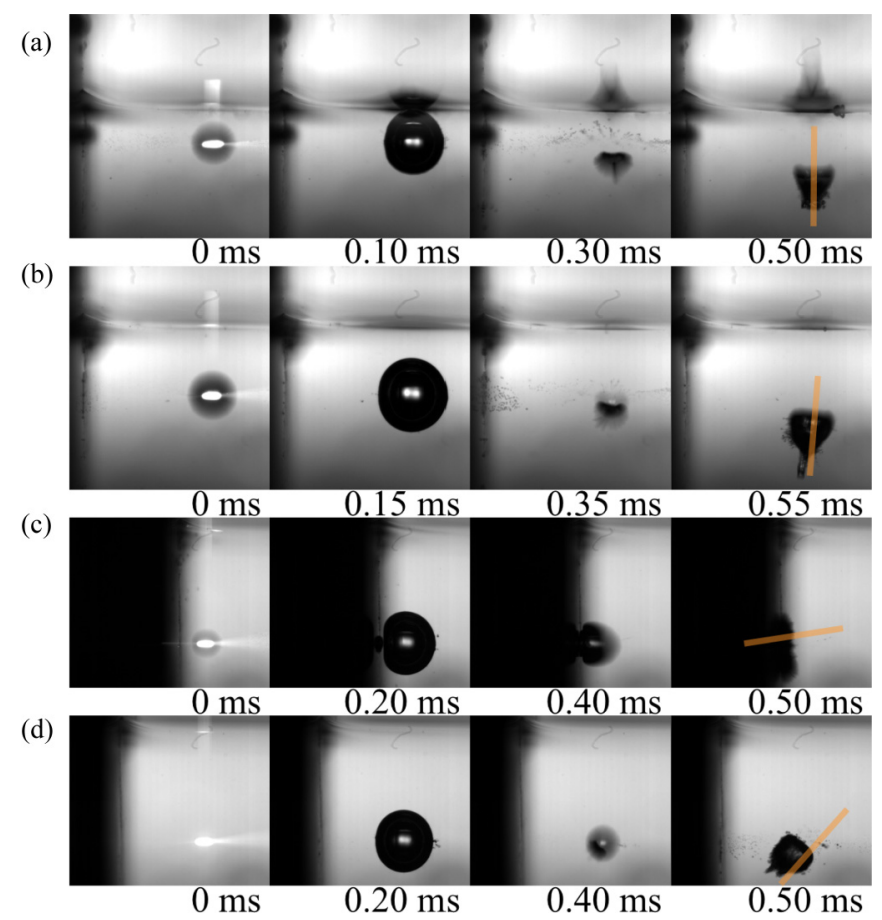

FIG. 6. A comparison of the cavitation behavior at different conditions (a)-(d). The approximate bubble locations were $h=2.1 \mathrm{~mm}, w=8.0 \mathrm{~mm}$, and $D=3.9 \mathrm{~mm}$ for (a), $h=4.1 \mathrm{~mm}, w=8.0 \mathrm{~mm}$, and $D=$ $4.4 \mathrm{~mm}$ for (b), $h=7.9 \mathrm{~mm}, w=2.2 \mathrm{~mm}$, and $D=4.5 \mathrm{~mm}$ for (c), and $h=7.9 \mathrm{~mm}, w=6.0 \mathrm{~mm}$, and $D=4.3 \mathrm{~mm}$ for (d). Orange lines on the last frame of the images show the approximate angle of the microjet predicted by the model. The solid wall is on the left-hand side of the bubble.

Figure 7 shows the behavior of a cavitation bubble at the corner $(\chi=0.85 \pm 0.06$ for $h \sim w \sim$ $2 \mathrm{~mm}$ and $\chi=0.92 \pm 0.02$ for $h \sim w \sim 3 \mathrm{~mm}$ ) with varying diameters $(2.0 \mathrm{~mm} \leqslant D \leqslant 4.7 \mathrm{~mm})$. A relatively small bubble at some distances from the boundaries [Fig. 7(a), $h / D \sim w / D \sim 1.5$ ] shows a smooth jet that the angle $\beta$ matches with the model prediction. The cavitation bubble maintains its shape as mostly spherical, as expected from the results shown in Fig. 6. Neither the deformation of the free surface nor the bubble onset at the boundaries was noticeable. Increasing the bubble diameter $D$ [Fig. 7(b)], and further decreasing the distances $h$ and $w$ [Fig. 7(c)], introduce the nonspherical cavitation bubble as well as the complex jet as discussed in Fig. 6. The deformation of the free surface, and bubble inception at the wall and corner [Figs. 7(b) and 7(c)], were noticeable. Especially in Fig. 7(c), the emerged cavities at the boundaries seemed to merge into the laser-induced cavitation bubble. However, we again note that the difference between the measured and predicted angles remains at a similar level to other results (see Fig. 5).

Figure 5 also shows the results from Fig. 1 using star symbols. The bubble shown in Fig. 1(b), located at $\chi \simeq 2.9$, produces a jet with $\beta \simeq 73^{\circ}$, a value which agrees well with the prediction in Eq. (2) (see an open star in Fig. 5). To define $\chi$ for the data shown in Fig. 1(a), we took the distance between the cavitation bubble center and the surface of the air bubble, $\lambda$, as the characteristic length, instead of $h$, where we find $\chi=\lambda / w \simeq 0.52$. Since the jet direction in Fig. 1(a) seems to be almost vertical, we consider $\beta \simeq 0^{\circ}$ (see a solid star in Fig. 5). The fair agreement suggests that our model could even be applied to the situation where the jet direction is modified by an air bubble instead of by the free surface. 
(a)

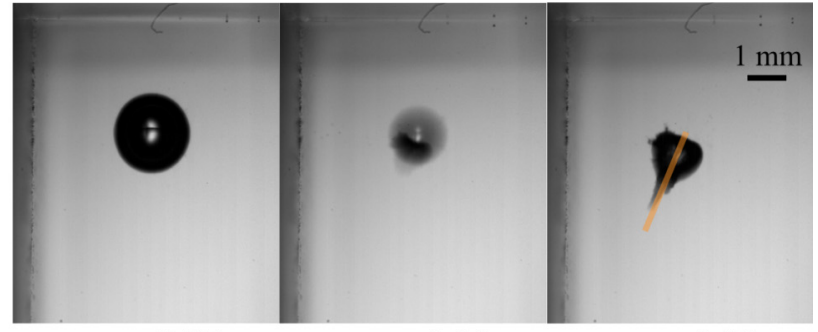

(b)

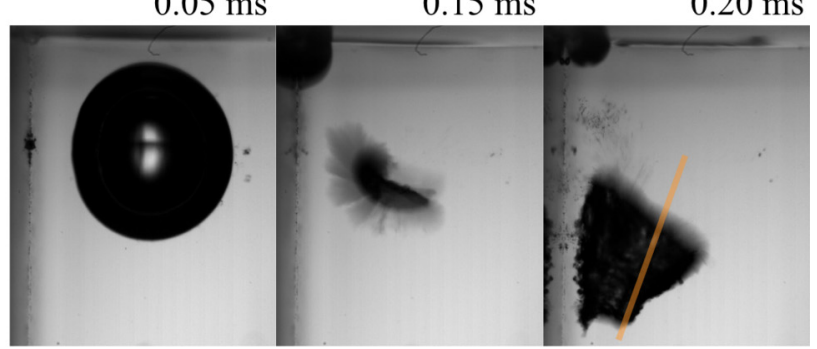

(c)

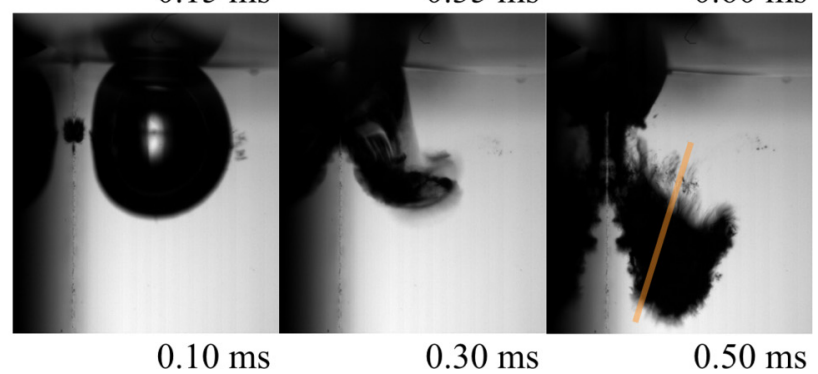

FIG. 7. A comparison of the cavitation behavior at the corner, where the diameter $D$ ranges from 2.0 to $4.7 \mathrm{~mm}$ (a)-(c). The approximate bubble locations were $h / D \sim w / D \sim 1.5$ for (a), $h / D \sim w / D \sim 0.6$ for (b), and $h / D \sim w / D \sim 0.5$ for (c). Orange lines on the last frame of the images show the approximate angle of the microjet predicted by the model. The solid wall is on the left-hand side of the bubble.

\section{AN EXTENSION TO VARIOUS $\alpha$ VALUES}

The approach of Tagawa and Peters [11] allows us to generalize the model for various $\alpha$ $[=\pi /(2 n)]$. As mentioned in the literature [11], the magnitude of the velocity vector $\vec{u}_{s}$ at the bubble located at $\overrightarrow{r_{s 0}}=l e^{i \theta_{s 0}}$ induced by, for example, a sink, located at $\vec{r}_{s}=l e^{i \theta_{s}}$ is expressed as $\left|\vec{u}_{s}\right|=Q /\left(4 \pi l^{2}\right)=Q /\left\{8 \pi l^{2}\left[1-\cos \left(\theta_{s 0}-\theta_{s}\right)\right]\right\}$, where $l$ is the distance between the sink and the center of the system $O$. The unit vector for the sink with respect to the bubble is expressed as $\left(e^{i \theta_{s}}-e^{i \theta_{s 0}}\right) /\left\{\sqrt{2\left[1-\cos \left(\theta_{s 0}-\theta_{s}\right)\right]}\right\}$. The general expression of the velocity vector can be obtained by multiplying those relationships as

$$
\vec{u}_{s}=\frac{Q}{\pi \sqrt{128} l^{2}} \frac{e^{i \theta_{s}}-e^{i \theta_{s 0}}}{\left[1-\cos \left(\theta_{s 0}-\theta_{s}\right)\right]^{3 / 2}} .
$$

The system for $n=1$ considered here [Fig. 8(a)] corresponds to that shown in Fig. 2. By applying the same strategy, we may place the sinks/sources for $n=2$ [i.e., $\alpha=\pi / 4\left(=45^{\circ}\right)$ ] as shown in Fig. $8(\mathrm{~b})$, or even higher $n$ values $[\alpha=\pi /(2 n)]$. The vector for the microjet $\vec{u}_{j}$ is given by summing 
(a)

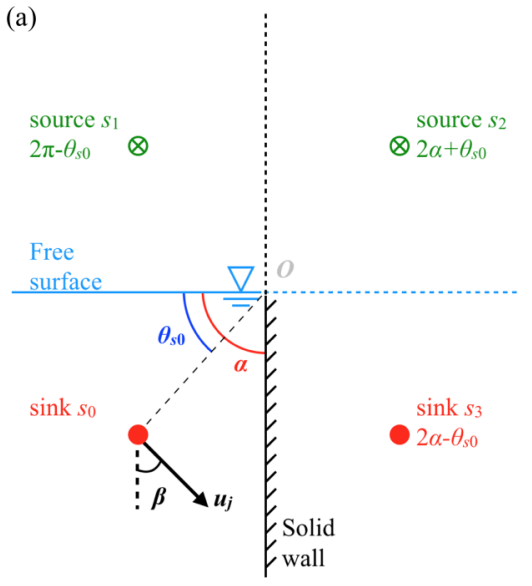

(b)

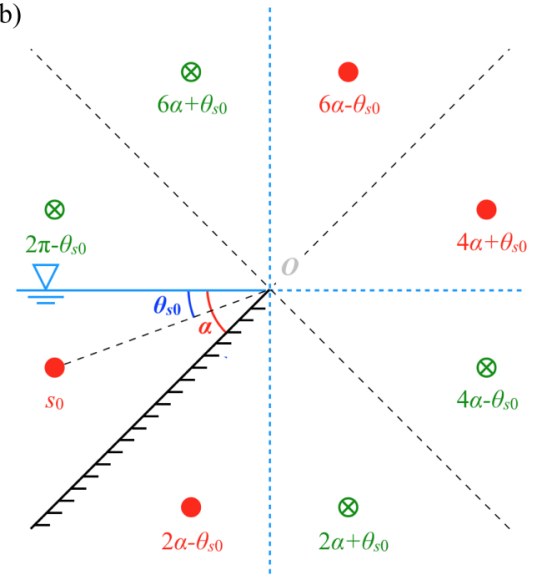

FIG. 8. The schematic of the system on the polar coordinates. Green markers $(\bigotimes)$ and red markers $(\bigcirc)$ respectively represent the sources and sinks, where $s_{0}$ represents the cavitation bubble. The corner opening angle $\alpha$ is $\pi / 2\left(=90^{\circ}\right)$ in (a) and $\pi / 4\left(=45^{\circ}\right)$ in (b). The sinks/sources in (a) are referred to as $s_{1}, s_{2}$, and $s_{3}$ for comparison purposes to Fig. 2. The angle of the image sinks/sources is denoted as a function of both the corner opening angle $\alpha$ and the bubble location $\theta_{s 0}$.

up all $\vec{u}_{s}$ belonging to the image sinks/sources, as

$$
\begin{aligned}
\frac{\vec{u}_{j}}{C}= & \frac{e^{i \theta_{s 0}}-e^{i\left(2 \pi-\theta_{s 0}\right)}}{\left[1-\cos \left(2 \theta_{s 0}\right)\right]^{3 / 2}} \\
& +\sum_{k=1}^{2 n-1}\left[\frac{e^{i \theta_{s 0}}-e^{i\left(2 k \alpha-\theta_{s 0}\right)}}{\left[1-\cos \left(2 \theta_{s 0}-2 k \alpha\right)\right]^{3 / 2}}-\frac{e^{i \theta_{s 0}}-e^{i\left(2 k \alpha+\theta_{s 0}\right)}}{[1-\cos (2 k \alpha)]^{3 / 2}}\right](-1)^{k} .
\end{aligned}
$$

The first term represents the influence of the induced flow by a source located at $\theta_{s}=2 \pi-\theta_{s 0}$, while the latter terms indicate those by the sinks/sources ( $n-1$ for each) located at $\theta_{s}=2 k \alpha \pm \theta_{s 0}$. Note that we assumed that all sinks/sources are located at the same distance $l$ from the center of the system $O$. The natural number $k$ represents the index from 1 to $2 n-1$. The prefactor $C$ in the equation above is expressed as $C=Q /\left(\pi \sqrt{128} l^{2}\right)$. The jet direction is thus obtained as

$$
\beta=\arctan \left(-\frac{\operatorname{Re}\left(\vec{u}_{j}\right)}{\operatorname{Im}\left(\vec{u}_{j}\right)}\right),
$$

without using the prefactor $C$.

Figure 8 shows the normalized jet angle $\beta / \alpha$ as a function of the location of the bubble $\theta_{s 0} / \alpha$ predicted by Eq. (7). We note that, for $n=1$ [i.e., $\alpha=\pi / 2\left(=90^{\circ}\right)$ ], the prediction matches with Eq. (2) (not shown). The well-defined nondimensional axes reveal the fact that increasing $n$ values (i.e., the finer corner angle $\alpha$ ) induces a slight shift of the curve as seen in the inset. However, even though we increase $n$ significantly, the influence of the first term in Eq. (7) remains in effect and the curve does not cross the point $\beta / \alpha=\theta_{s 0} / \alpha=0.5$. It perhaps belongs to the same reason as to why Eq. (2) predicts $\beta=45^{\circ}$ not at $\chi=1$ but $\chi \simeq 1.38$, meaning that this is a unique feature of such a system made by two different boundaries; the free surface has a relatively dominant effect on the jet direction $\beta$ when compared to the solid wall. Though the situation for $\alpha<\pi / 2$ is a bit difficult to test experimentally and thus speculative, our extension of the previous work [11] to our system might deepen our understanding of the jet formation upon the cavity collapse at the complex geometry. 


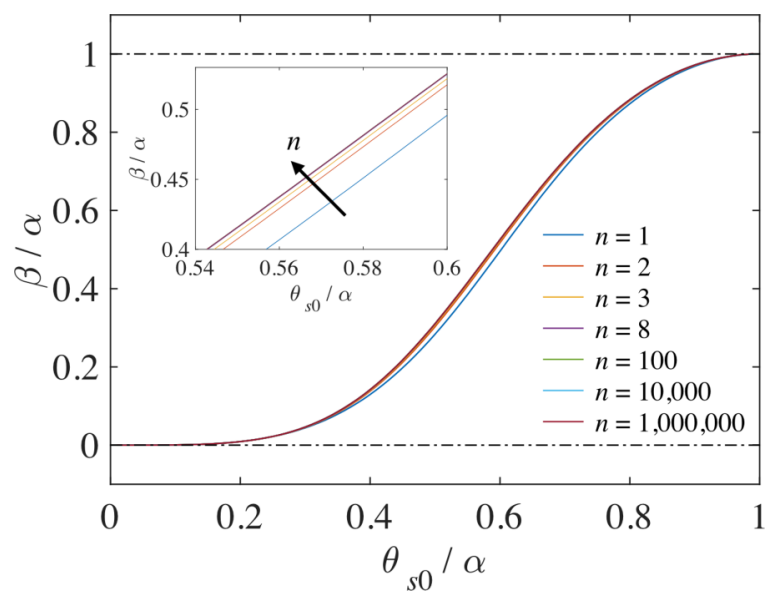

FIG. 9. The predictions of the jet angle $\beta / \alpha$ as a function of the bubble position $\theta_{s 0} / \alpha$ calculated by Eq. (7) with varying $n$ values. A zoom-in view is shown in the inset. Dashed lines indicate $\beta / \alpha=0$ and $\beta / \alpha=1.0$.

\section{CONCLUSION}

We investigated the direction $\beta$ of the microjet caused by a cavitation bubble collapsing in a corner of a wall and free surface. We derived the theoretical prediction for the jet angle deflection $\beta$ as a function of the bubble location parameter $\chi=h / w$ [Eq. (2)] based on the sink-source system (Fig. 2). We compared the model predictions with our systematic experiment (Fig. 4), finding a fair agreement between the calculated and measured values (see Fig. 5). The model is even potentially applicable to the case where the jet is influenced by an air bubble (Fig. 1). We note that the model might not be applicable to describe the cases when the air bubble is located in between the cavitation bubble and the wall, or the wall is covered by bubbles [19]. In addition, we extended the approach presented by Tagawa and Peters [11] to our system and derived the equation that predicts the jet direction $\beta$ caused by the bubble at a corner with an angle, $\alpha=\pi /(2 n)$ [see Eq. (7)], where the case for $n=1$ leads to the same result as that from Eq. (2). A numerical calculation with sufficiently large $n$ values revealed that the free surface has a relatively dominant effect on the microjet direction upon the cavitation collapse when compared to the solid wall (Fig. 9).

For future studies, though the prediction of the jet direction might be a hint to a practical design, it does not necessarily guarantee the prevention of cavitation-induced damage. To estimate the strength of the damage caused by the microjet, the impact velocity of the jet to the wall also needs to be calculated. In addition, other possible sources of the damage (i.e., the shockwave that propagates upon the rebound of cavitation bubble) also need to be taken into account. Future research is thus warranted.

\section{ACKNOWLEDGMENTS}

We thank T. Yamaguchi for his support in the experiments. A.K. is thankful for support as a JSPS Overseas Research Fellow now at Utah State University. This project is supported by the Institute of Global Innovation Research in TUAT for J.M.G.'s summer stay at TUAT. Y.T. acknowledges financial support from JSPS KAKENHI Grants No. 17H01246 and No. $20 \mathrm{H} 00223$.

[1] M. S. Plesset and R. B. Chapman, Collapse of an initially spherical vapour cavity in the neighbourhood of a solid boundary, J. Fluid Mech. 47, 283 (1971). 
[2] C.-T. Hsiao, A. Jayaprakash, A. Kapahi, J. K. Choi, and G. L. Chahine, Modelling of material pitting from cavitation bubble collapse, J. Fluid Mech. 755, 142 (2014).

[3] E. A. Brujan, G. S. Keen, A. Vogel, and J. R. Blake, The final stage of the collapse of a cavitation bubble close to a rigid boundary, Phys. Fluids 14, 85 (2002).

[4] Y. Tomita, P. B. Robinson, R. P. Tong, and J. R. Blake, Growth and collapse of cavitation bubbles near a curved rigid boundary, J. Fluid Mech. 466, 259 (2002).

[5] P. Cui, A.-M. Zhang, S. Wang, and B. C. Khoo, Ice breaking by a collapsing bubble, J. Fluid Mech. 841, 287 (2018).

[6] E. A. Brujan, K. Nahen, P. Schmidt, and A. Vogel, Dynamics of laser-induced cavitation bubbles near elastic boundaries: influence of the elastic modulus, J. Fluid Mech. 433, 283 (2001).

[7] Y. Tomita and T. Kodama, Interaction of laser-induced cavitation bubbles with composite surfaces, J. Appl. Phys. 94, 2809 (2003).

[8] P. Gregorčič, R. Petkovšek, and J. Možina, Investigation of a cavitation bubble between a rigid boundary and a free surface, J. Appl. Phys. 102, 094904 (2007).

[9] A. Kucera and J. R. Blake, Approximate methods for modelling cavitation bubbles near boundaries, Bull. Aust. Math. Soc. 41, 1 (1990).

[10] E.-A. Brujan, T. Noda, A. Ishigami, T. Ogasawara, and H. Takahira, Dynamics of laser-induced cavitation bubbles near two perpendicular rigid walls, J. Fluid Mech. 841, 28 (2018).

[11] Y. Tagawa and I. R. Peters, Bubble collapse and jet formation in corner geometries, Phys. Rev. Fluids 3, 081601(R) (2018).

[12] R. H. Cole, Underwater Explosions (Princeton University Press, Princeton, NJ, 1948).

[13] Q. Wang, M. Mahmud, J. Cui, W. R. Smith, and A. D. Walmsley, Numerical investigation of bubble dynamics at a corner, Phys. Fluids 32, 053306 (2020).

[14] E.-A. Brujan, H. Takahira, and T. Ogasawara, Planar jets in collapsing cavitation bubbles, Exp. Therm. Fluid Sci. 101, 48 (2018).

[15] L. Molefe and I. R. Peters, Jet direction in bubble collapse within rectangular and triangular channels, Phys. Rev. E 100, 063105 (2019).

[16] E. D. Andrews, D. F. Rivas, and I. R. Peters, Cavity collapse near slot geometries, J. Fluid Mech. 901, A29 (2020).

[17] C. Brennen, Cavitation and Bubble Dynamics (Oxford University Press, Oxford, UK, 1995).

[18] J. Luo, W.-1. Xu, and R. Li, Collapse of cavitation bubbles near air bubbles, J. Hydrodyn. 32, 929 (2020).

[19] S. R. Gonzalez Avila, D. M. Nguyen, S. Arunachalam, E. M. Domingues, H. Mishra, and C.-D. Ohl, Mitigating cavitation erosion using biomimetic gas-entrapping microtextured surfaces (GEMS), Sci. Adv. 6, eaax6192 (2020).

[20] J. R. Blake and D. C. Gibson, Cavitation bubbles near boundaries, Annu. Rev. Fluid Mech. 19, 99 (1987).

[21] J. R. Blake and P. Cerone, A note on the impulse due to a vapour bubble near a boundary, J. Aust. Math. Soc., Ser. B: Appl. Math. 23, 383 (1982).

[22] Y. Tagawa, S. Yamamoto, K. Hayasaka, and M. Kameda, On pressure impulse of a laser-induced underwater shock wave, J. Fluid Mech. 808, 5 (2016).

[23] O. Supponen, D. Obreschkow, M. Tinguely, P. Kobel, N. Dorsaz, and M. Farhat, Scaling laws for jets of single cavitation bubbles, J. Fluid Mech. 802, 263 (2016). 